\title{
Thermal design studies in superconducting rf cavities: Phonon peak and Kapitza conductance
}

\author{
A. Aizaz, ${ }^{1, *}$ T. L. Grimm, ${ }^{2}$ and N. T. Wright ${ }^{3}$ \\ ${ }^{1}$ Department of Aerospace Engineering, CAE National University of Sciences and Technology, Risalpur, Pakistan \\ ${ }^{2}$ Niowave Inc., East Lansing, Michigan 48906, USA \\ ${ }^{3}$ Department of Mechanical Engineering, Michigan State University, East Lansing, Michigan 48823, USA
}

(Received 1 February 2010; published 22 September 2010)

\begin{abstract}
Thermal design studies of superconducting radio frequency (SRF) cavities involve two thermal parameters, namely the temperature dependent thermal conductivity of $\mathrm{Nb}$ at low temperatures and the heat transfer coefficient at the Nb-He II interface, commonly known as the Kapitza conductance. During the fabrication process of the SRF cavities, $\mathrm{Nb}$ sheet is plastically deformed through a deep drawing process to obtain the desired shape. The effect of plastic deformation on low temperature thermal conductivity as well as Kapitza conductance has been studied experimentally. Strain induced during the plastic deformation process reduces the thermal conductivity in its phonon transmission regime (disappearance of phonon peak) by $80 \%$, which may explain the performance limitations of the defect-free SRF cavities during their high field operations. Low temperature annealing of the deformed $\mathrm{Nb}$ sample could not recover the phonon peak. However, moderate temperature annealing during the titanification process recovered the phonon peak in the thermal conductivity curve. Kapitza conductance measurements for the $\mathrm{Nb}-\mathrm{He}$ II interface for various surface topologies have also been carried out before and after the annealing. These measurements reveal consistently increased Kapitza conductance after the annealing process was carried out in the two temperature regimes.
\end{abstract}

\section{INTRODUCTION}

Niobium $(\mathrm{Nb})$ made superconducting radio frequency (SRF) cavities are used to accelerate charged particles to near the speed of light. These cavities have much lower losses than those made of copper, thus allowing much higher average fields.

One phenomenon that limits the theoretical achievable fields is thermal breakdown of superconductivity. In thermal breakdown, the temperature of part or the entire rf surface exceeds the critical temperature, thereby becoming normal conducting and rapidly dissipating all of the stored energy in the cavity fields. Practically, there are two kinds of thermal breakdowns: thermal breakdown due to material defects and the thermal breakdown due to global thermal instability (GTI).

Material defects are the most commonly understood cause of thermal breakdown in SRF cavities. It has been shown in previous studies [1] that the defect in the $\mathrm{Nb}$ skin is thermally isolated from the cooling effects of the surrounding liquid helium and the breakdown of the magnetic field is totally dependent only upon the temperature dependent thermal conductivity of the bulk $\mathrm{Nb}$ at temperatures above $4 \mathrm{~K}$.

The second type of thermal breakdown, usually attributed to high surface magnetic field regions, is GTI [1]. In GTI, the heating is nearly uniform over all of the high

\footnotetext{
*Corresponding author. aizazahm@msu.edu
}

surface magnetic field regions. GTI occurs because, even without localized defects, a portion of the superconducting rf surface resistance depends on the rf surface temperature. Under these circumstances, losses, such as surface resistance $R_{s}$ which has exponential temperature dependence as shown in Eq. (1), ultimately limit the maximum achievable gradients in the SRF cavity,

$$
R_{s}=A \frac{\omega^{2}}{T_{s}} \exp \left(-\frac{\Delta(o)}{k_{B} T_{s}}\right)+R_{o} .
$$

The first term on the right-hand side of Eq. (1) is the BCS resistance. For temperatures less than $T_{c} / 2$, the binding energy $\Delta(0)$ is nearly unchanged from its value at absolute zero. The coefficient $A$ is a complex function of material parameters such as the superconducting coherence length, the penetration depth, the electron mean-free path, and the Fermi velocity. The $\omega$ is frequency, $k_{\mathrm{B}}$ is the Boltzmann constant, and $T_{s}$ is the rf surface temperature. The BCS resistance in a typical $3 \mathrm{GHz}$ cavity varies from $3 \mu \Omega$ at $T=4.2 \mathrm{~K}$ to less than $1 \mathrm{n} \Omega$ at $T=1.4 \mathrm{~K}$.

The second term on the right-hand side of Eq. (1) is the residual resistance $R_{o}$, which is temperature independent. Mechanisms for $R_{o}$ are not well understood, though several possibilities have been proposed and investigated $[2,3]$. Residual resistance values are generally found to be between 5 and $100 \mathrm{n} \Omega$, though values as low as $1 \mathrm{n} \Omega$ have been measured [4].

As the advancements take place with technology, high residual resistance ratio (RRR) $\mathrm{Nb}$ sheets for SRF cavity fabrication are now becoming routine. Thus, with im- 


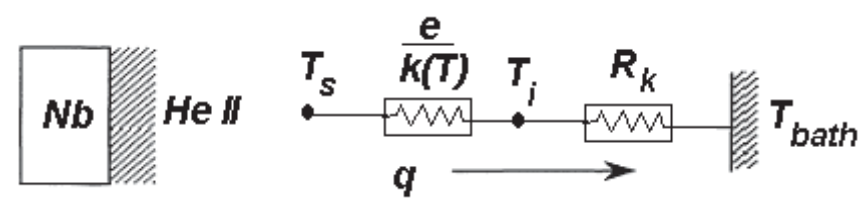

FIG. 1. Thermal circuit of a defect-free SRF cavity in a pool of helium at bath temperature of $T_{\text {bath }} . T_{s}$ is the rf surface temperature of $\mathrm{Nb}$ cavity with thickness $e$ and having temperature dependent thermal conductivity $k(T) . T_{i}$ is the intermediate temperature representing outer surface temperature and $R_{k}$ is the Kapitza resistance when the helium is in the superfluid phase.

proved purity in $\mathrm{Nb}$ material, a case of near defect-free cavity, the possibility of thermal breakdown due to GTI becomes more likely during the high field measurements. It is in this situation that the phonon peak in the thermal conductivity of the $\mathrm{Nb}$ plays a significant role in determining the quench field of the SRF cavity.

For the case of a defect-free cavity, the effect of Kapitza resistance $R_{k}$ or $\left(1 / h_{k}\right)$ and the thermal conductivity $k$ on the accelerating field $E_{\text {acc }}$ can be investigated based upon a simple 1D heat transfer model [5], as shown in Fig. 1.

Consider that the heat flux $q$ in $\mathrm{W} / \mathrm{m}^{2}$ during steady operation is solely produced by Joule heating at the inner surface wall having temperature $T_{s}$ and is uniquely due to the rf surface resistance $R_{s}$. Then it can be expressed as

$$
q=\frac{1}{2 \mu_{o}^{2}} R_{s} B^{2},
$$

where $\mu_{o}$ is the magnetic permeability, $B$ expressed in Tesla is the magnetic flux density on the rf surface of the cavity, and $R_{s}$ is the surface resistance in ohms, which itself is a function of $\mathrm{rf}$ surface temperature $T_{S}$ as given in Eq. (1). Also, from the thermal circuit of Fig. 1, assuming constant properties, heat flux can be correlated with thermal conductivity and the Kapitza resistance,

$$
q=\left[\frac{1}{e / k+R_{k}}\right]\left(T_{s}-T_{\text {bath }}\right) .
$$

Thermal breakdown occurs when the rf surface of the niobium reaches its critical temperature $T_{c}$ which is dependent on the critical rf magnetic field $B_{c}$ (expressed in $\mathrm{mT}$ ) by

$$
T_{c}(K) \approx 9.2\left(1-\frac{B_{c}}{200}\right)^{1 / 2} \text {. }
$$

\section{THERMAL-MAGNETIC INTERACTIONS}

The relative importance of $k$ and $h_{k}$ on the thermal stability can be estimated for the case of a defect-free SRF cavity. The maximum achievable magnetic fields can be obtained by solving Eqs. (2) and (3) iteratively while using Eq. (1) for the definition of surface resistance $R_{s}[5]$.

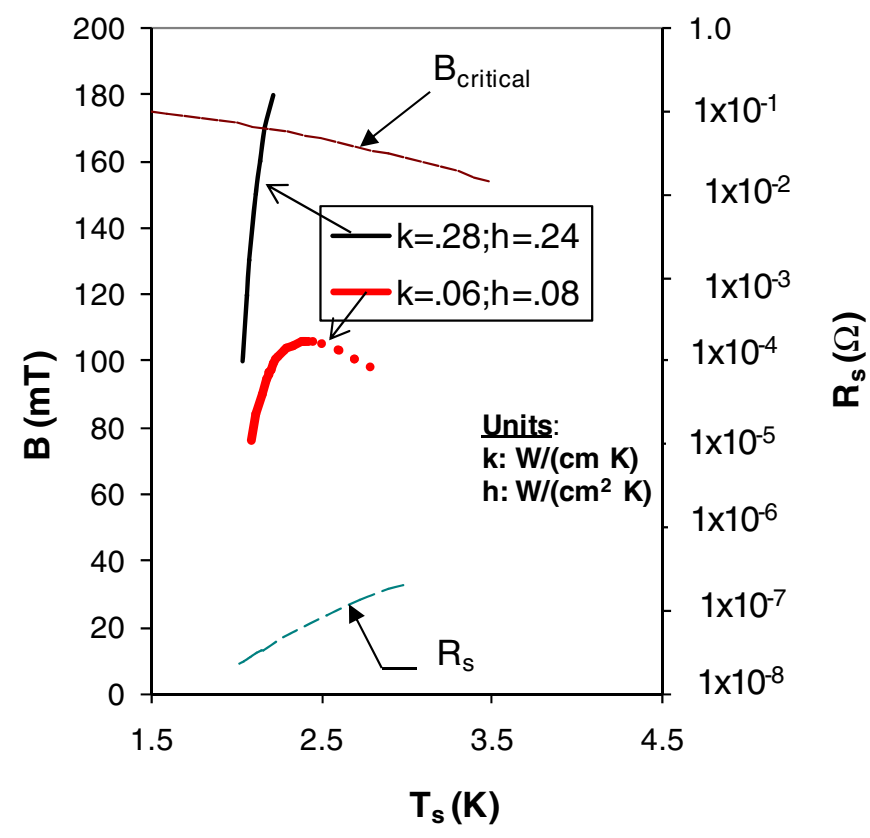

FIG. 2. (Color) Thermal-magnetic numerical estimates with constant thermal properties, $k$ and $h$, for the two cases are shown. About 5 times increase in $k$ and 3 times increase in $h$ resulted in almost $50 \%$ improvement in applied magnetic fields. Parameters used for these numerical estimates are $R_{\text {res }}=5 \mathrm{n} \Omega, e=3 \mathrm{~mm}$, $T_{b}=2 \mathrm{~K}, f=1.3 \mathrm{GH} z$, and $\mathrm{RRR}=230$. The rise in surface resistance (right axis) due to rf surface temperature increase is also plotted.

In Fig. 2, the magnetic field $(B)$ is plotted as a function of the rf surface temperature for the two sets of values of thermal conductivity and Kapitza conductance. The maximum achievable magnetic field for the $k=$ $0.28 \mathrm{~W} /(\mathrm{cm} \mathrm{K})$ case reached nearly to $170 \mathrm{mT}$. However, for the other case $(k=0.06 \mathrm{~W} /(\mathrm{cm} \mathrm{K}))$, the maximum achievable magnetic field is not more than $110 \mathrm{mT}$. Note is to be made in this case that the dotted thick line past the maxima is due to the quadratic nature of Eq. (2) and reflects a nonphysical solution [5].

These constant property numerical estimates clearly show the effect of increased $k$ and $h_{k}$ on the higher allowable magnetic field, which is then limited by the critical magnetic field [Eq. (3)] only. Another important observation which can be made from these numerical estimates is that, for a defect-free cavity (a case for $k=$ $0.28 \mathrm{~W} / \mathrm{cm} \mathrm{K}$ ), the maximum rf surface temperature rise due to applied magnetic fields is on the order of a few hundred $\mathrm{mK}$ only. Thus, the constant property approximation in making the numerical estimates is not a bad choice. This observation also helps in understanding the operating range of temperature dependent thermal conductivity of $\mathrm{Nb}$, as explained in the next section.

While the thermal conductivity above $\sim 4 \mathrm{~K}$ is well known and correlated with RRR, for temperatures below $\sim 3 \mathrm{~K}$ the correlation is lost due to phonon conduction. In 
this temperature regime, the microstructure of the material plays an important role in determining the thermal conductivity of $\mathrm{Nb}$. Significant changes in microstructure take place when the SRF cavities are routinely fabricated through plastically deforming the metal during the deep drawing process. The effect of plastic deformation on the thermal conductivity of $\mathrm{Nb}$ and on its Kapitza conductance is the focus of the present study.

\section{EFFECT OF THERMAL CONDUCTIVITY ON SRF CAVITIES}

At the operating temperatures of the cavity, when $\mathrm{Nb}$ is in its superconducting state, its thermal conductivity varies dramatically with temperature and is governed by the interplay of both of the heat carriers, i.e., electrons and phonons. The thermal conductivity of $\mathrm{Nb}$ above $4 \mathrm{~K}$ is dominated by the electronic conduction of thermal energy. For temperatures below $\sim 3 \mathrm{~K}$, due to dominant phonon conduction, the microstructure of the bulk $\mathrm{Nb}$, characterized by strains and lattice imperfections or grain size and grain boundaries, plays a vital role in determining the final shape of the thermal conductivity curve. Though not always the case with high RRR Nb material, a typical curve for large grain (through annealing) $\mathrm{Nb}$ thermal conductivity usually shows local maxima below $\sim 3 \mathrm{~K}$, known as "phonon peak" [1].

Much emphasis has been placed in the past on the techniques for improving thermal conductivity of the bulk $\mathrm{Nb}$ for temperatures above $\sim 4 \mathrm{~K}$. This was done to reduce the effect of "localized defects" on the performance of the SRF cavities [1]. However, for the defect-free cavities, as has been discussed earlier, the total rf surface temperature rise is on the order of few hundreds of $\mathrm{mK}$. Thus, the phonon thermal conduction regime $(<3 \mathrm{~K})$ of $\mathrm{Nb}$ plays the dominant role in determining the quench field of defect-free high frequency cavities operating at bath temperatures less than $2.1 \mathrm{~K}$. Nevertheless, for both of the cases, i.e., high frequency defect-free cavities $(<3 \mathrm{~K})$ or the cavities with defects $(>4 \mathrm{~K})$, thermal conductivity of $\mathrm{Nb}$ remains an important parameter for the improvement of cavities performance. Annealing the $\mathrm{Nb}$ at temperatures above $\sim 1400^{\circ} \mathrm{C}$ in a high vacuum furnace creates large grain size resulting in the formation of a "phonon peak" in the thermal conductivity of $\mathrm{Nb}$ [1].

\section{EFFECT OF KAPITZA CONDUCTANCE ON SRF CAVITIES}

The other resistive parameter in the thermal circuit of Fig. 2 is the $R_{k}$, i.e., Kapitza resistance to heat transfer through the liquid He-II. As mentioned above, in the case of defects on the rf surface of the cavity, the heat transfer coefficient at the $\mathrm{Nb}-\mathrm{He}$ interface does not play as strong a role as the thermal conductivity in determining the quench field. This may be the reason that little attention has been given in the past to this particular aspect for the improvement of performance in SRF cavities. However, the importance of the heat transfer to the pool bath of liquid helium increases, as the thermal conductivity improves with purity, or as the $\mathrm{Nb}$ wall thickness is decreased, or if there are no defects as illustrated through simple thermal-magnetic numerical estimates presented in Fig. 2. Therefore it seems quite logical to explore various aspects of improving Kapitza conductance, for the improvement of the cavity performance.

\section{EXPERIMENTAL TEST SAMPLES}

Test results from two types of samples, i.e., cylinders and flat rectangular plates, are presented here. The cylindrical samples, $\mathrm{S} 1$ and $\mathrm{S} 2$, were taken from a long $\mathrm{Nb}$ rod of small grain supplied by Tokyo Denkai. The supplier reported a RRR value of 232 for the ingot of supplied $\mathrm{Nb}$. Measurements of both the thermal conductivity and the Kapitza conductance are reported for these two cylindrical samples. Wah Chang supplied the two rectangular flat plate samples, F1 and F2, for thermal conductivity measurements to evaluate the possibility of partial crystallization in the "as-received" $\mathrm{Nb}$. The supplier for these samples reported a RRR value of 300 .

\section{A. Cylindrical sample preparation}

A series of tests were conducted on the two $\mathrm{Nb}$ cylindrical samples, to evaluate the effect of various surface and bulk conditions. Table I lists the treatments of each test performed on these samples where test \#1 precedes others in chronological order. Sample conditions that may have an effect on the bulk material properties, such as plastic deformations and heat treatment or annealing, their thermal conductivity results are analyzed. The $\mathrm{Nb}$-He interface surface conditions are also analyzed that affect the Kapitza conductance, such as BCP etching, mechanical polishing, surface roughness, and annealing. Here, the term "BCP" stands for buffer chemical polish (1:1:2), a chemical etching procedure routinely employed on SRF cavities to remove surface contaminations and improve surface texture. The rough surface of sample S2, as shown in Fig. 3, can be qualitatively compared with a relatively smoother surface of sample S1. Also, titanification is a gettering process usually employed on the SRF cavities to improve the purity of the $\mathrm{Nb}$. This should result in an increased RRR value of the $\mathrm{Nb}$ by removing the interstitial impurities. Since the process takes place at moderate temperatures of $\sim 1300^{\circ} \mathrm{C}$ to $1400^{\circ} \mathrm{C}$, the grain size of the $\mathrm{Nb}$ also increases significantly. Subsequently, the outer contaminated surface, $\sim 45$ to $50 \mu \mathrm{m}$, is removed through regular $\mathrm{BCP}$ etching.

\section{B. Flat plate $\mathrm{Nb}$ sample preparation}

Both flat plate samples are identical to each other in the as-received condition and have undergone the same heat 
TABLE I. Surface and bulk preparation of the two cylindrical niobium samples.

\begin{tabular}{|c|c|c|c|}
\hline Test \# & Sample & Surface & Process \\
\hline 1 & $\mathrm{~S} 1$ & Flat and clean surface & Machine cut and light BCP etch $(10-20 \mu \mathrm{m})$ \\
\hline 1 & $\mathrm{~S} 2$ & Flat surface & Machine cut \\
\hline 2 & $\mathrm{~S} 1$ & Flat smooth & Mechanical. polish to a mirrored surface and light BCP etch $(5-6 \mu \mathrm{m})$ \\
\hline 2 & $\mathrm{~S} 2$ & Rough $\sim$ of $1-2 \mathrm{~mm}$ & $\begin{array}{l}\text { Cut surface with stainless steel knife edge through mechanical hand } \\
\text { press deep enough for SI }>3 \text {. Light BCP etch }(5-6 \mu \mathrm{m})\end{array}$ \\
\hline 3 & $\mathrm{~S} 1$ & Flat smooth & $\begin{array}{l}\sim 3 \% \text { of total length plastically deformed axially and mechanical polish } \\
\text { to mirrored surface and light BCP etch }(10-15 \mu \mathrm{m})\end{array}$ \\
\hline 3 & $\mathrm{~S} 2$ & Rough $\sim$ of $1-2 \mathrm{~mm}$ & $\mathrm{BCP}$ etch cleaning and heat treatment at $750^{\circ} \mathrm{C}$ for $2 \mathrm{hrs}$ \\
\hline 4 & $\mathrm{~S} 1$ & Flat smooth & $\begin{array}{l}\text { Mirror polished and titanification at } 1300^{\circ} \mathrm{C} \\
\text { for } 2 \mathrm{hrs} \text { and } 1200^{\circ} \mathrm{C} \text { for } 4 \mathrm{hrs} \text { and } \mathrm{BCP} \text { etch }(\sim 50 \mu \mathrm{m})\end{array}$ \\
\hline 4 & $\mathrm{~S} 2$ & Rough $\sim$ of $1-2 \mathrm{~mm}$ & Titanification at $1300^{\circ} \mathrm{C}$ for $2 \mathrm{hrs}$ and $1200^{\circ} \mathrm{C}$ for $4 \mathrm{hrs}$ and $\mathrm{BCP}$ etch $(\sim 50 \mu \mathrm{m})$ \\
\hline
\end{tabular}

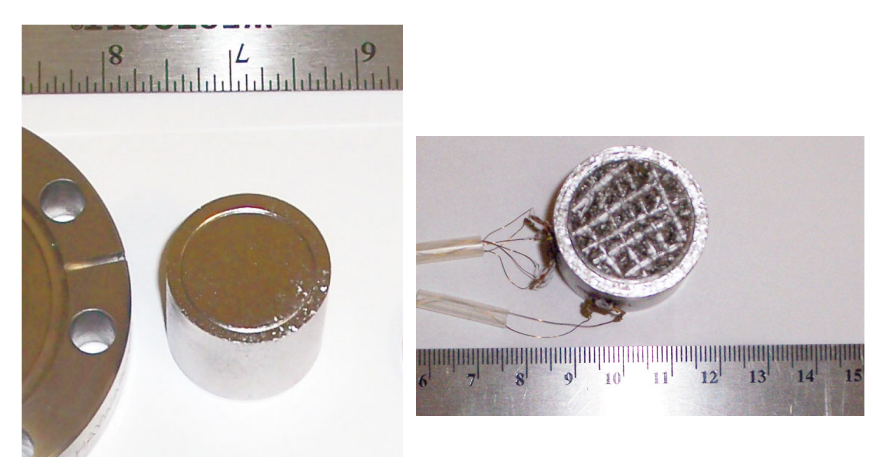

FIG. 3. Two cylindrical samples S1 (left view) and S2 (right view) are shown. The S2 sample has a rough surface $(\sim 1-2 \mathrm{~mm})$ as compared with the smooth surface of sample $\mathrm{S} 1$.

treatment $\left(750^{\circ} \mathrm{C}\right.$ for 2 hours) process for test $\# 6$ as that for cylindrical sample S2 in test \#3. Table II lists the preparation details of these samples prior to each test.

\section{EXPERIMENTAL SETUP}

The cylindrical $\mathrm{Nb}$ samples $(3 \mathrm{~cm}$ diameter by $3 \mathrm{~cm}$ long) are mounted on the sample holder, as shown in Fig. 4, and placed in an evacuated chamber assembly. Three in situ calibrated carbon sensors, attached along the length of each sample, are used to measure the temperature rise in the sample when a dc heat load is applied to its one end through a thin foil heater. The other end of the sample is exposed to liquid helium ( $\mathrm{LHe}$ ) contained in a stainless steel tube, as shown in Fig. 4.
This experimental arrangement has the advantage of measuring both the thermal conductivity as well as the $\mathrm{Nb}-\mathrm{LHe}$ interface heat transfer coefficient in the same experimental run. The interface temperature is estimated by linear extrapolation. Details of the experimental setup and error estimation analysis are presented in [6].

For the flat plate samples, the sample holder assembly, as shown in Fig. 5, is simply a blank conflat flange. A right angle bend is made at one end of the flat plate sample to attach the sample to the flange using a brass screw.

The heater on the other end of the sample provides the desired input heat load. Using the same evacuated chamber with an arrangement of three collinear carbon sensors, similar to those for the cylindrical samples, the measurements for thermal conductivity of these flat plate rectangular samples are made. The total error estimate in the measurements of thermal conductivity from both the experimental arrangements and the Kapitza conductance measurements is less than $10 \%$ [6].

\section{THERMAL CONDUCTIVITY MEASUREMENTS}

\section{A. Establishing baseline}

The repeatability of the experimental result, validation with the literature data, and the error estimation has been published elsewhere [6]. Here, thermal conductivity measurements of $\mathrm{Nb}$ sample $\mathrm{S} 1$ and $\mathrm{S} 2$ in test \#1 are shown in Fig. 6 for the bath temperature of $2.0 \mathrm{~K}$. Below $\sim 3 \mathrm{~K}$, the hump in thermal conductivity is clearly visible where the maxima occurring at $\sim 2 \mathrm{~K}$, as discussed earlier, is known

TABLE II. Bulk preparation of two flat plate niobium samples.

\begin{tabular}{lcll}
\hline \hline Test \# & Sample & Surface & Process \\
\hline 5 & F1 & Flat smooth & Ordinary ethanol cleaning to remove dirt/grease marks \\
5 & F2 & Flat smooth & Ordinary ethanol cleaning to remove dirt/grease marks. \\
6 & F1 & Flat smooth & BCP etch cleaning and heat treatment at $750^{\circ} \mathrm{C}$ for 2 hrs \\
6 & F2 & Flat smooth & BCP etch cleaning and heat treatment at $750^{\circ} \mathrm{C}$ for 2 hrs \\
\hline \hline
\end{tabular}




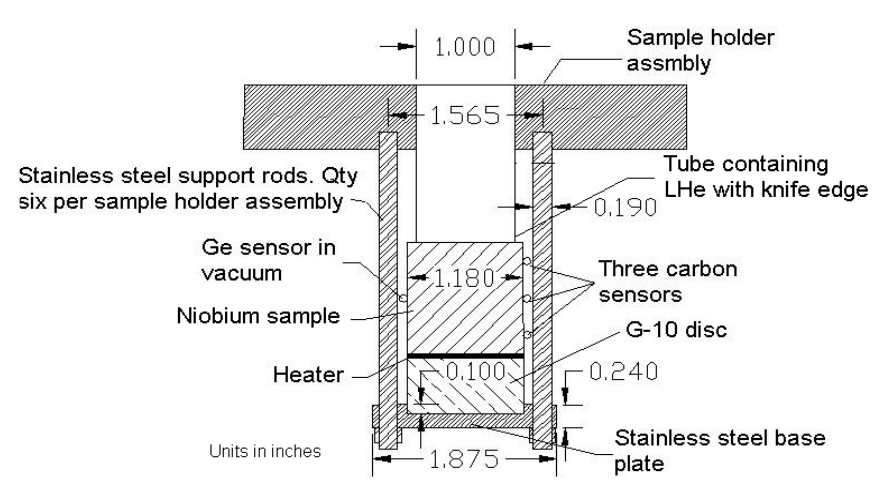

FIG. 4. Cylindrical Nb sample experimental setup. The sample holder assembly has a $\mathrm{Nb}$ sample with a heater on the bottom side and a stainless steel tube with knife edge containing liquid helium on the other side.

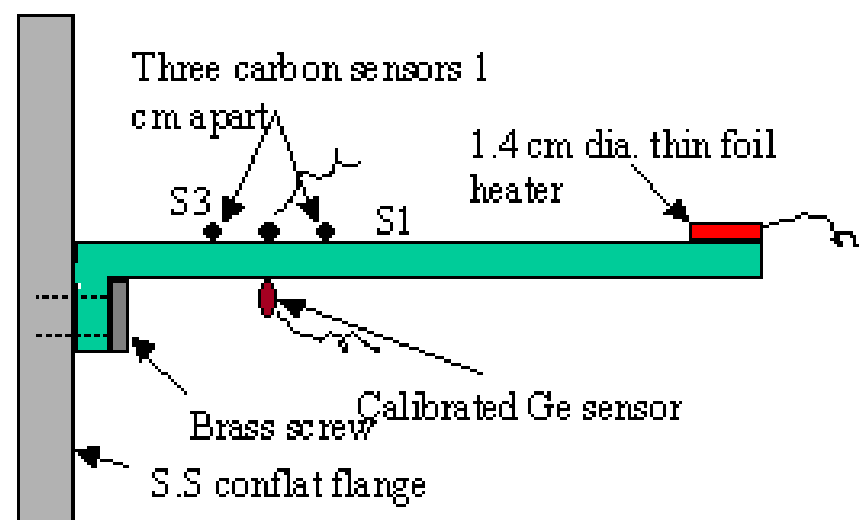

FIG. 5. (Color) Flat plate experimental setup. The Nb sample of size $11 \times 1.4 \times 0.3 \mathrm{~cm}$ has a germanium sensor on one side and three carbon sensors on the other, for in situ calibrations. The drawing is not to scale.

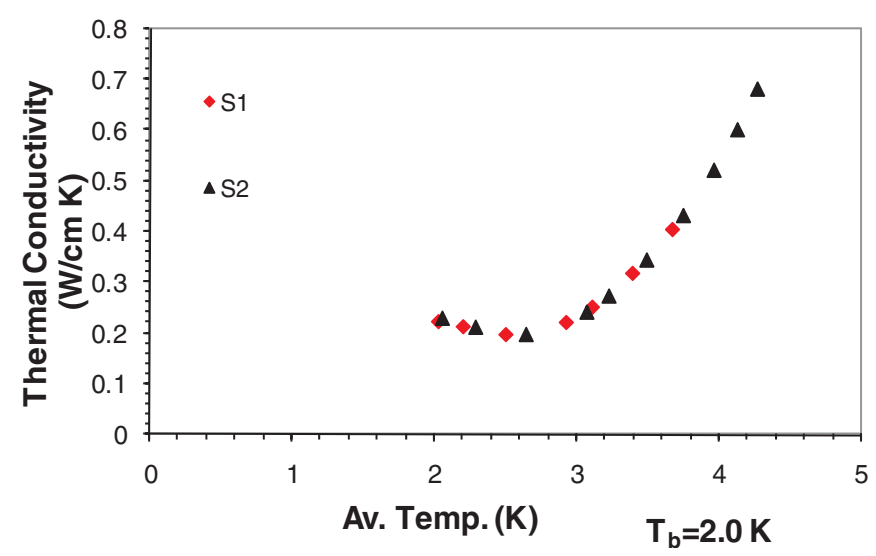

FIG. 6. (Color) The phonon peak is shown near average temperature of $2 \mathrm{~K}$ in thermal conductivity measurements of the two samples, $\mathrm{S} 1$ and $\mathrm{S} 2$, in test \#1 $\left(T_{b}=2 \mathrm{~K}\right)$. as "phonon peak." As expected, there is no significant difference in the thermal conductivity measurements of the two samples as both are from the same bulk material.

\section{B. A new trend in the data \\ 1. Effect of plastic deformations}

Measurements of the thermal conductivity in tests \#2 and \#3 are compared in Fig. 7 for sample S1. As noted from Table I, for test \#3, sample S1 was intentionally compressed axially to induce a nominal axial strain $(\Delta L / L)$ of $\sim 3 \%$ in the sample because of plastic deformation. The absence of a phonon peak and a nearly $80 \%$ reduction in thermal conductivity at $2 \mathrm{~K}$ in test \#3 for $\mathrm{S} 1$, in contrast with the values obtained in test \#2, are considered to be the effect of plastic deformation on the thermal conductivity of $\mathrm{Nb}$. As expected, this effect is insignificant at temperatures above $\sim 3 \mathrm{~K}$, where the electronic contribution to thermal conductivity of $\mathrm{Nb}$ starts to dominate and no significant difference in the results from the two tests is evident.

\section{Effect of low temperature heat treatment}

For sample S2 in Fig. 8, the absence of a phonon peak in test \#2, in contrast with those of test \#1 in Fig. 7, can be understood as a surface roughness preparation protocol, as described in Table I and the picture shown in Fig. 3, with the stainless steel blade pressing against the surface, causing inadvertent strains in the bulk $\mathrm{Nb}$, similar to those induced intentionally in S1.

Low temperature annealing $\left(750^{\circ} \mathrm{C}\right.$ for $\left.2 \mathrm{hrs}\right)$ on $\mathrm{S} 2$, intended to recover the phonon peak in the $\mathrm{Nb}$ and plotted in Fig. 8, is insufficient though the S2 demonstrates a small increase $(\sim 30 \%)$ in thermal conductivity over the temperature range measured. This is possibly due to knocking off some of the hydrogen impurities at the annealing

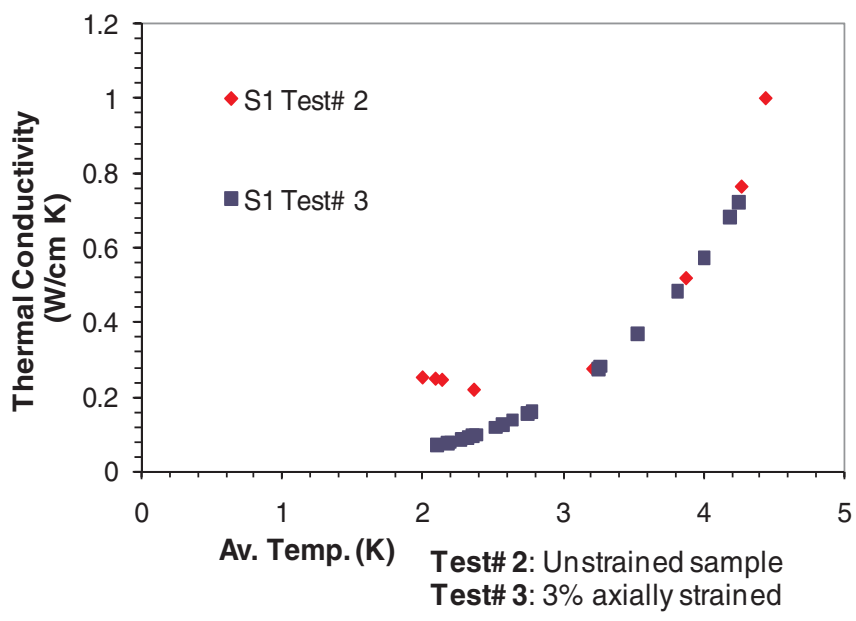

FIG. 7. (Color) The phonon peak is clearly absent in the thermal conductivity measurements of sample $\mathrm{S} 1$ in the tests carried out after $3 \%$ strains induced in the sample (test \#3) as compared with its measurement in test \#2. 


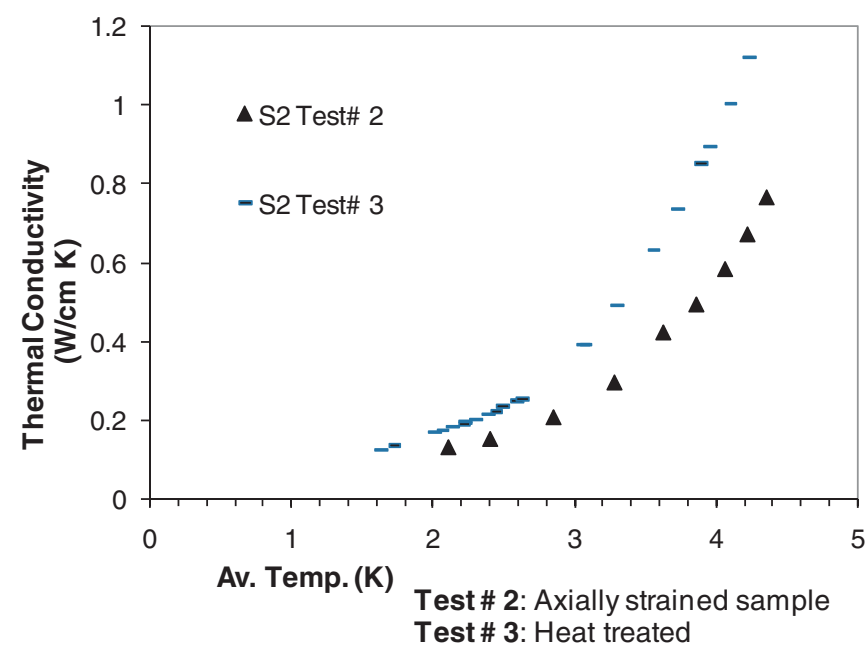

FIG. 8. (Color) The phonon peak did not reappear after it was lost during the compression process in preparation of (test \#2) sample S2. The low temperature heat treatment (test \#3) has a small effect on the thermal conductivity of the niobium.

temperatures causing a slight increase in the RRR value of the sample.

The same effect of low temperature annealing is also observed in thermal conductivity measurements done on the two flat plate samples and shown in Fig. 9. The initial measurements on flat samples done in test \#5 did not reveal a phonon peak in the as-received material, possibly due to partial recrystallization in the material. After annealing them at $750^{\circ} \mathrm{C}$ for 2 hours, no significant change in their thermal conductivities was observed for most of the temperature range measured. However, a slight improvement below $\sim 3 \mathrm{~K}$ can still be observed due to the low temperature heat treatment.

The result for the sample F1 is presented in Fig. 9(a) and those of F2 is in Fig. 9(b). Close agreement of these results increases the confidence in the experimental measurements.

Based on these observations, it can be argued that low temperature annealing is insufficient to recover the lost phonon peak in the thermal conductivity curve of $\mathrm{Nb}$ with RRR ranging from 200 to 300 .

\section{Effect of titanification}

During the titanification process, the temperature of the sample is raised to $\sim 1300^{\circ} \mathrm{C}$ in high vacuum conditions. At these moderate temperatures, sufficient annealing within the $\mathrm{Nb}$ takes place to relieve internal strains in the material. Also, the grain size of $\mathrm{Nb}$ grows from the order of $\mu \mathrm{m}$ to $\mathrm{mm}$. As shown in Fig. 10 for sample S1 and Fig. 11 for sample S2, this process resulted in the recovery of phonon peaks in the thermal conductivity for the two $\mathrm{Nb}$ samples that were lost due to induced strains. There is slightly more recovery of phonon peak for sample S2 after titanification than it was in the $\mathrm{S} 1$, the as-received sample.

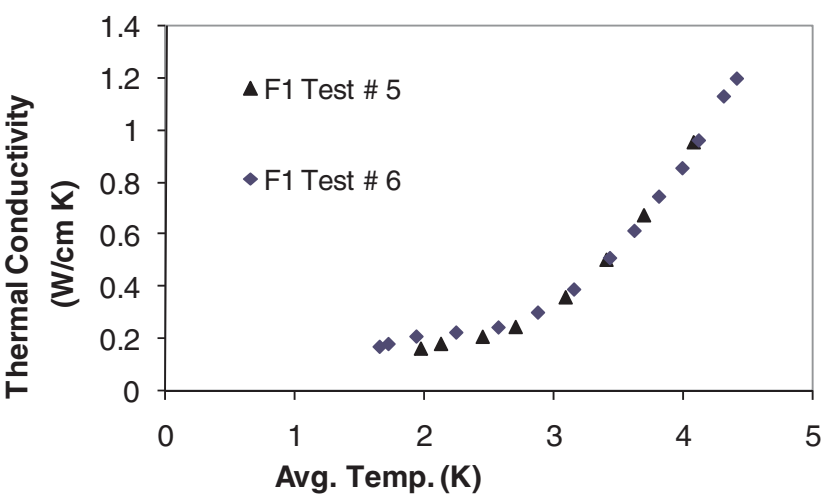

(a)

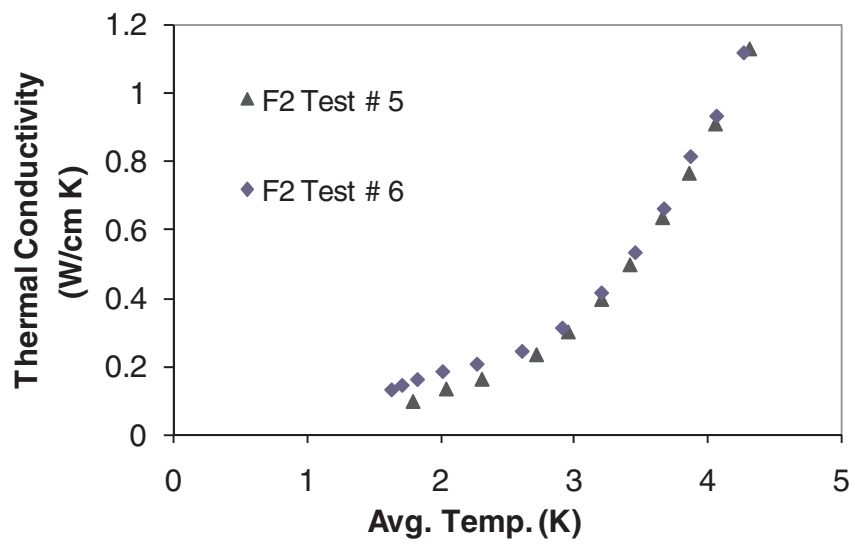

(b)

FIG. 9. (a),(b): The low temperature heat treatment (test \#6) has slightly increased the thermal conductivity of flat samples in the phonon conduction regime (below $3 \mathrm{~K}$ ), from their previous values in test \#5 on the as-received samples.

This could be due to the fact that sample S2 had already undergone low temperature annealing with a slight increase in its thermal conductivity below $3 \mathrm{~K}$, as mentioned

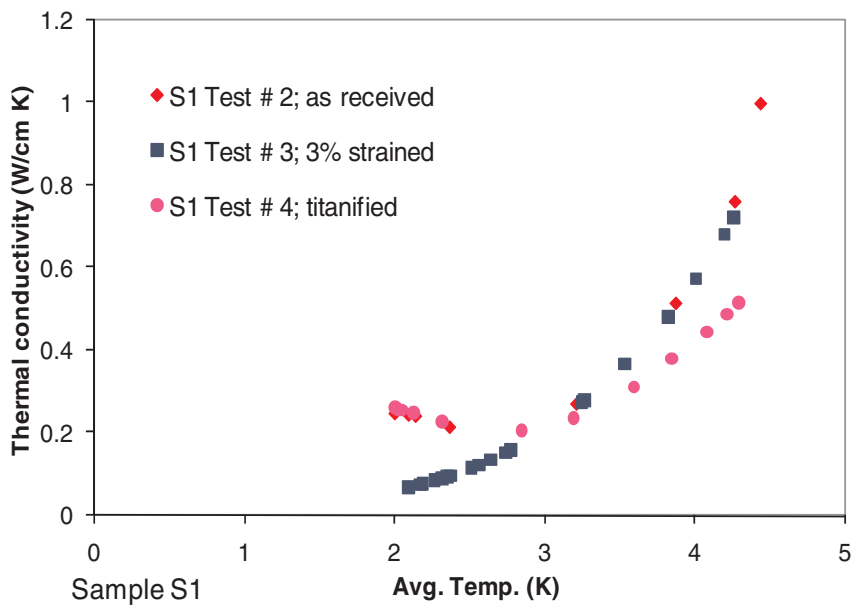

FIG. 10. (Color) Moderate temperature annealing during the titanification process resulted in the reformation of the phonon peak (test \#4) after it was lost due to induced strains (test \#3) in the as-received $\mathrm{Nb}$ sample $\mathrm{S} 1$ (test \#2). 


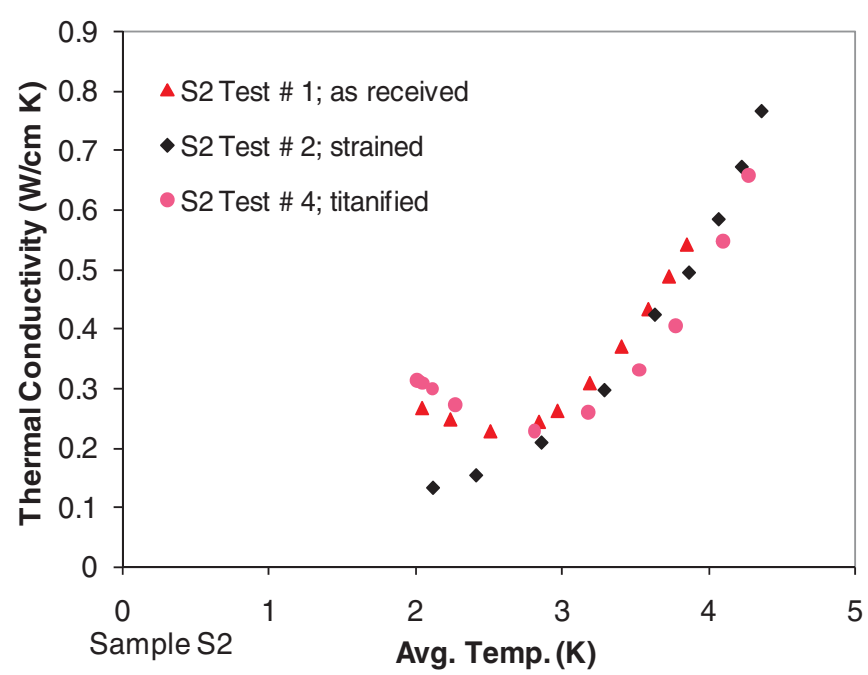

FIG. 11. (Color) Moderate temperature annealing during titanification resulted in the reformation of phonon peak (test \#4) after it was lost due to inadvertent strains induced (test \#3) in the asreceived $\mathrm{Nb}$ sample $\mathrm{S} 2$ (test \#1).

in Sec. II above. Possibly, this second annealing at moderate temperatures augmented the reformation of a phonon peak slightly more than it was in the as-received sample.

Unexpectedly, a slight decrease in the thermal conductivity above $\sim 3 \mathrm{~K}$ is also noticeable in both samples, $\sim 20 \%$ in sample 1 and $\sim 8 \%$ in sample 2 at $4.2 \mathrm{~K}$, as compared to their values before annealing. This is understandable since the purification process through titanification relies on the diffusion of oxygen molecules in the bulk to the surface of niobium where they are captured by titanium. The diffusion rate of oxygen at these temperatures is $\sim 1 \mathrm{~mm}$ per hour [7], and the titanification process is optimized to treat 2 to $3 \mathrm{~mm}$ thick $\mathrm{Nb}$ cavities. However, the cylindrical samples have $30 \mathrm{~mm}$ diameter. It seems quite reasonable that a much longer time than the 6 hours, namely $(15 \mathrm{~mm} / 1(\mathrm{~mm} / \mathrm{hr})) 15 \mathrm{hrs}$, for the cylindrical samples were needed for complete purification.

\section{KAPITZA CONDUCTANCE MEASUREMENTS}

Several studies reveal that nanometer length scale surface roughness plays an important role in affecting the Kapitza conductance [8,9]. At a macroscopic level, larger scale roughness carries more number of smaller scale roughness superimposed onto them. Thus, increased surface index (SI) of the surface may lead to enhanced Kapitza conductance. Here SI refers to the ratio of actual surface area of a rough surface to that of its projected area.

\section{A. Establishing baseline}

The BCP etched sample S1, having a cleaner surface than S2, has Kapitza conductance at $2 \mathrm{~K}$ that is about 2 times that of S2, as shown in Fig. 12, for test \#1. The

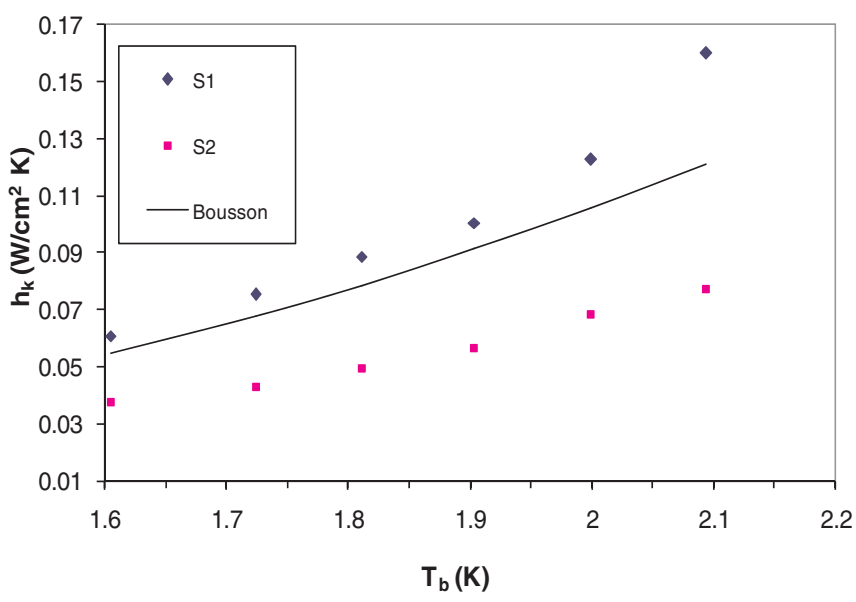

FIG. 12. (Color) Kapitza conductance measurements for the two cylindrical samples, $\mathrm{S} 1$ and $\mathrm{S} 2$, carried out in test \#1, are compared with the one given by Bousson et al. [10].

Kapitza conductance measurements of S1 are in reasonable agreement with the values provided in the literature by Bousson et al. [10] for $\mathrm{Nb}$ having comparable RRRs and from the same supplier.

\section{B. Repeatability}

The measurement of Kapitza conductance of the sample S1 before and after plastic deformation, as shown in Fig. 13, does not reveal any significant difference. This is likely due to the surface preparation for the two tests being identical on sample S1, i.e., mechanical polish and a light $\mathrm{BCP}$ etch.

\section{Effect of surface index}

In order to evaluate the effect of large SI on Kapitza conductance, the surface roughness of sample S2 was increased such that its surface index became greater than

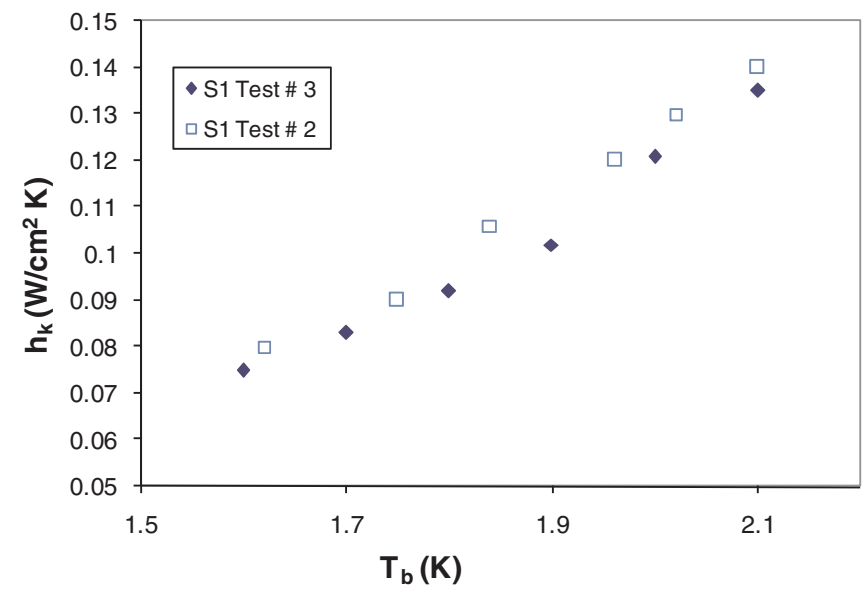

FIG. 13. (Color) Kapitza conductance measurements of the sample S1 before and after the plastic deformations reveal no significant difference. 


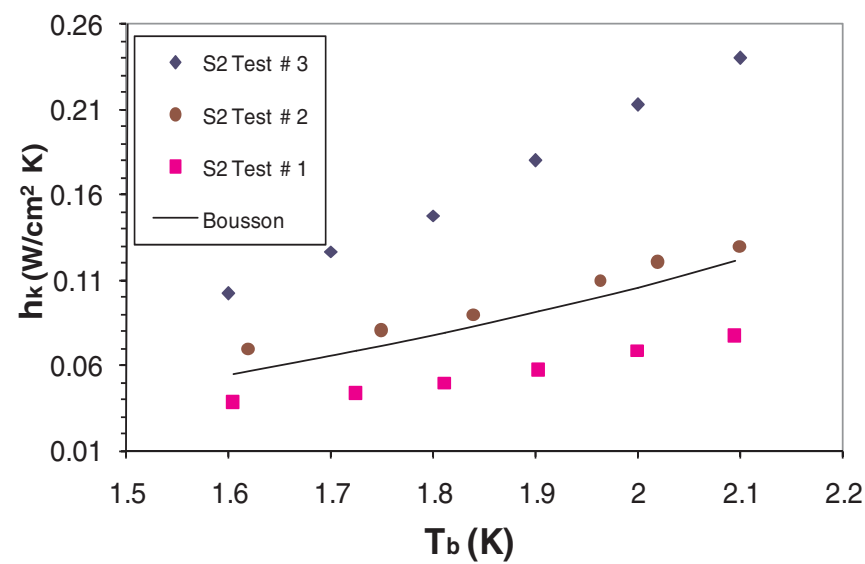

FIG. 14. (Color) The Kapitza conductance of the sample S2 surface with SI $>3$ (test \#2) and the effect of low temperature annealing with SI $>3$ (test \#3) is compared with the machine cut surface (test \#1). Literature data is adapted from Bousson et al. [10].

3. A sharp stainless steel edge surface was pressed against the $\mathrm{Nb}$ with the help of a hand press. Several deep (1$2 \mathrm{~mm}$ ) cuts both in a horizontal as well as in vertical directions, like a 2D grid on a flat surface and shown in Fig. 3, resulted in an overall SI $>3$. Results of Kapitza measurements of sample S2 as shown in Fig. 14 can be compared for its test \#2 (SI > 3) with those of its test \#1 (flat machine cut surface). Although there is $\sim 64 \%$ increase in $h_{k}$ in test \#2 measurements from its test \#1 values and this brought them closer to the data by Bousson et al. [10], however, this is still below the values expected proportionately to SI $>3$. One possible explanation could be the effect of surface strains on the Kapitza conductance that might have been inadvertently induced on the surface during the roughness process for $\mathrm{S} 2$.

The slight increase in $h_{k}$ for $\mathrm{S} 2$ in test 2 from test 1 can possibly be due to the BCP etching of the surface that took place for the preparation of test \#2 and brought the values of sample S2 closer to the literature data and the data of sample S1 for its test \#1 as presented in Fig. 13.

\section{Effect of low temperature annealing}

The same sample surface on S2, after annealing (test \#3) at $750^{\circ} \mathrm{C}$ for 2 hours, resulted in nearly doubling its Kapitza conductance values at $2 \mathrm{~K}$, as shown in Fig. 14 . This result may provide some help in understanding the effect of low temperature annealing on the Kapitza conductance by relieving some of the induced surface strains during the process.

\section{E. Effect of moderate temperature annealing}

The moderate temperature annealing employed during the titanification process relieves the stresses not only inside the $\mathrm{Nb}$ but also on the surface. This surface effect on the Kapitza conductance is shown in Fig. 15 for sample S1

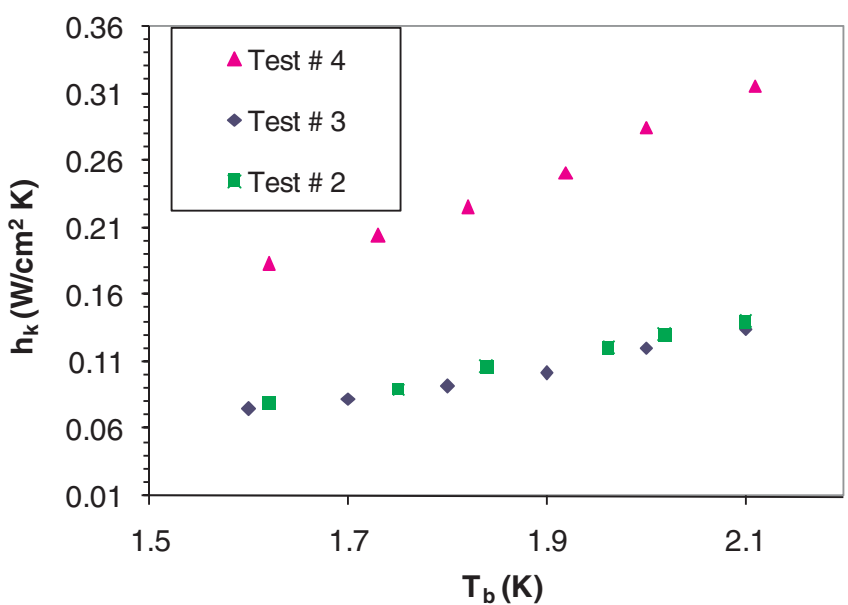

FIG. 15. (Color) Kapitza conductance for sample S1 comparison before (tests \#2 and \#3) and after titanification (test \#4).

and in Fig. 16 for sample S2. The greater than twofold increase in Kapitza conductance at $2 \mathrm{~K}$ for sample $\mathrm{S} 1$ from its test \#3 is possibly due to the moderate temperature annealing that occurred during the titanification process. For sample S2, the increase in Kapitza conductance is about $\sim 30 \%$ as compared to its test \#3 when its value was already increased through low temperature annealing discussed above.

If the increase in Kapitza conductance is compared for the two samples together, as in Fig. 17, it is seen that after the titanification process both samples (S1 and S2 in test \#4) attained comparably close values for the Kapitza conductance. This is despite the fact that both samples have surface roughness drastically different from each other. It is recalled from Fig. 3 that sample $\mathrm{S} 1$ has a smooth and plane surface whereas sample $\mathrm{S} 2$ has a rough surface (on the order of $\sim 1-2 \mathrm{~mm}$ ) resulting in $\mathrm{SI}>3$. Although the increase for sample $\mathrm{S} 2$ (in test \#4) is about 4 times from its initial state (test \#1), this result suggests that surface effects

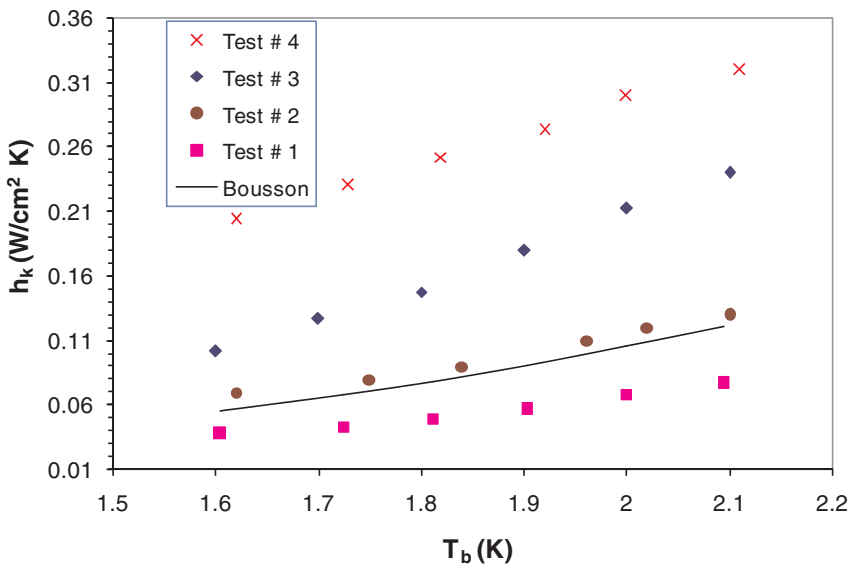

FIG. 16. (Color) Kapitza conductance increase history for sample S2 through all the processes especially after titanification (test \#4). 


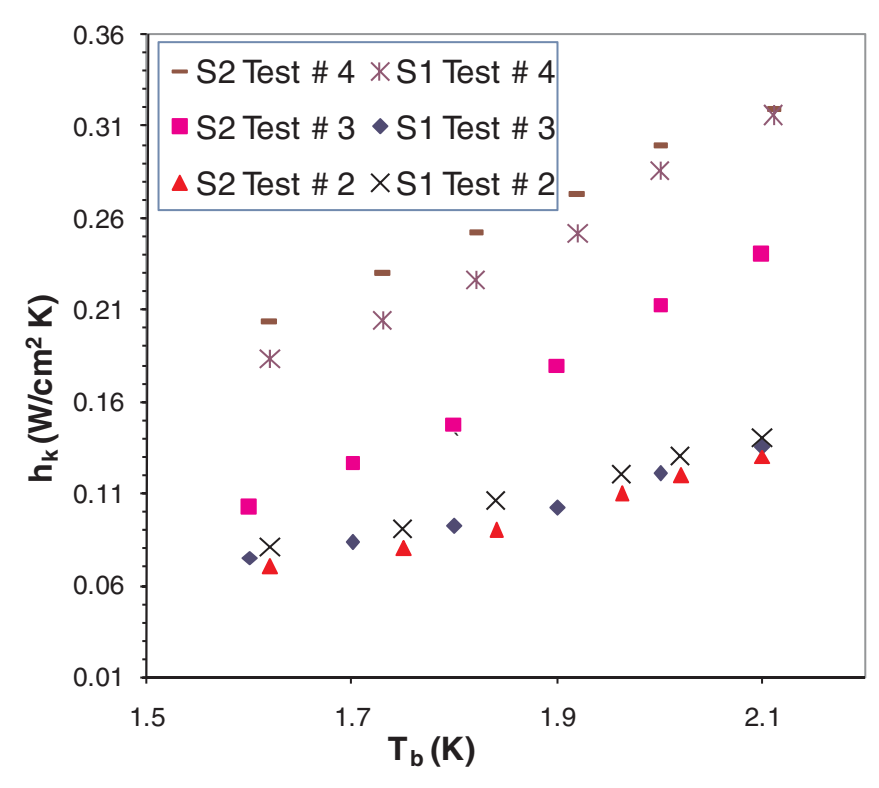

FIG. 17. (Color) Comparison of Kapitza conductance values of the two samples, S1 and S2, after titanification (test \#4).

due to titanification could be stronger on increasing the Kapitza conductance of the $\mathrm{Nb}$ samples than from the simple area increase. The exact nature of these effects that resulted in the increase of Kapitza conductance after titanification is still undetermined. Surface strains relieved during moderate temperature annealing in titanification process could be one possible reason. Other factors that may result in this unexpected result could be from the distribution of crystal orientation $(\langle 111\rangle$ or $\langle 110\rangle)$ on the surface. The crystal size growth during the titanification process may result in a substantially different distribution of crystal orientation closer to the surface as compared to their distribution before the titanification process. If the distribution of crystal orientations on the surface were more random than a uniform distribution of the same crystal orientation, the overall surface would be rough at the scale lengths of nanometer, the scale lengths, which are also comparable to the phonon wavelengths of He-II at these temperatures. This understanding may help explain the observation of comparably closer Kapitza conductance values for the two samples after titanification despite their apparent differences in surface roughness at the macro level. Nevertheless, this is the future work required for the subsequent studies in completely understanding the Kapitza conductance.

\section{DISCUSSION}

Several test for the thermal conductivity and Kapitza conductance measurements have been carried out. Thermal conductivity measurements are made on two different shapes of $\mathrm{Nb}$ samples, i.e., cylinder and flat plate. These measurements show not only close agreement with each other but also with the already published literature data to authenticate the results both for their repeatability as well as validity. At temperatures below $2.17 \mathrm{~K}$, i.e., below $T_{\lambda}$ when helium is in the superfluid state, Kapitza conductance measurements are done on two cylindrical samples S1 and S2 for various surface conditions.

\section{A. Thermal conductivity}

Although the thermal conductivity of $\mathrm{Nb}$ above $4 \mathrm{~K}$ is well correlated with its RRR value, the same is not true when the temperature is below $3 \mathrm{~K}$. As discussed earlier, it is in this range of temperatures when the thermal conductivity of $\mathrm{Nb}$ is dominantly governed by phonon conduction. Thus, the history of microstructure of the material becomes important. Higher purity (high RRR) Nb does not necessarily mean presence of phonon peak in the thermal conductivity at $2.0 \mathrm{~K}$. Even a small amount of plastic deformations ( $\sim 3 \%$ of strains) induced at any stage of material processing can cause the loss of phonon peak, as demonstrated in this research. This results in a significant reduction (about $80 \%$ at $2 \mathrm{~K}$ ) of the thermal conductivity. This is especially important when the SRF cavities are routinely fabricated through the deep drawing process in which plastic deformations are deliberately induced to shape the cavity to the design requirements. Moderate temperature annealing, as demonstrated in this research, is a viable remedy to recover back the lost phonon peak in order to achieve higher gradients in the SRF cavities.

The major advantage of the improved phonon peak in the thermal conductivity is for high frequency defect-free cavities operating at $2 \mathrm{~K}$. To realize the significance of the phonon peak on the improved performance of SRF cavities, recall Fig. 2 that was presented in the Introduction to this paper. Recall that the numerical estimates in this figure are with constant thermal parameters, i.e., constant thermal conductivity and the Kapitza conductance.

To a first order approximation, the numerical estimates shown in Fig. 2 provide a good performance indicator for SRF cavities with lost phonon peak (thick line such as for unannealed or plastically deformed $\mathrm{Nb}$ ) as compared with the recovered phonon peak (upper curve thin line such as annealed $\mathrm{Nb}$ ). Also note that the corresponding values of Kapitza conductance so chosen for the numerical estimates have also been duly demonstrated in this experimental study, which are close to the two cases of Kapitza conductance, i.e., before and after moderate temperature annealing. The numerical estimate reveals that the applied magnetic field rose from 105 to $170 \mathrm{mT}$ and only limited by the $T_{C}$. Thus ignoring other effects such as high field $Q$ slope, $\sim 50 \%$ improvement in the applied magnetic field is realizable due to the presence of phonon peak and improved Kapitza conductance. Moreover, both of the objectives, namely the phonon peak as well as improved Kapitza conductance, can be attained through one single process of moderate temperature annealing of the $\mathrm{Nb}$ cavities. 
For future research, moving forward in this direction, it is possible that a much higher phonon peak is realizable than the one demonstrated in this research either through high temperature annealing ( $\sim 1800$ to $2000^{\circ} \mathrm{C}$ ), which may have a drawback of considerable reduction in the yield strength of $\mathrm{Nb}$, or for longer durations of annealing at moderate temperatures. Also, beside other factors, since it is the strains in the microstructure of the $\mathrm{Nb}$ which determine the presence of the phonon peak, it would be interesting to analyze the effect of unstrained single crystal/large grain $\mathrm{Nb}$ material on the size of phonon peak as compared with the polycrystalline $\mathrm{Nb}$. Not to limit here, the effect of annealing of large grain/single crystal material may also be a fruitful option to explore.

\section{B. Kapitza conductance}

The study has revealed a significant effect of annealing on the improvement of Kapitza conductance. At least a $150 \%$ increase in the Kapitza conductance has been demonstrated in this study at $2.1 \mathrm{~K}$ due to moderate temperature annealing. As discussed earlier, the effect of increased SI on Kapitza conductance does not seem to play a strong role as compared with the effect of annealing of the samples. Among other factors, possibly removal of surface strains that might have been induced during the roughness process, has resulted in the increased Kapitza conductance after annealing. Also, since it is the nanolength scale roughness that is more important, crystal orientations on the surface could be one possible parameter that have changed due to annealing.

The effect of improved Kapitza conductance on the performance of SRF cavities has already been discussed in the above section while discussing the importance of phonon peak in the thermal conductivity of $\mathrm{Nb}$. Also note that, as the thermal conductivity of $\mathrm{Nb}$ is improved due to enhanced phonon peak, the importance of Kapitza conductance on the performance of SRF cavities becomes even more prominent. Even with such a substantial improvement of Kapitza conductance after annealing has been demonstrated, yet the data found in this study for the Kapitza conductance is on the lower end of the wide spread of the data available in the literature for $\mathrm{Nb}$. Hence, room for further improvement in the Kapitza conductance still exists. Also, further study is required to point out the exact parameter that changed during the process of annealing and resulted in the improvement of Kapitza conductance.

\section{CONCLUSIONS}

\section{A. Effect of phonon peak in Nb thermal conductivity}

This study revealed that the strains induced through plastic deformations reduced the thermal conductivity of $\mathrm{Nb}$ by $80 \%$ at $2 \mathrm{~K}$.

This resulted in the loss of phonon peak. Low temperature annealing (at $750^{\circ} \mathrm{C}$ for 2 hours) was found to be insufficient to recover the lost phonon peak. However, titanification done at $\sim 1300^{\circ} \mathrm{C}$ restored the lost phonon peak near to its as-received values. Ignoring other effects, simple 1D numerical computations performed in this study reveal that the effect of recovery of the lost phonon peak along with improved Kapitza conductance resulted in $\sim 50 \%$ improvement in the applied magnetic fields for the case of $1.3 \mathrm{GHz}$ cavity operating at $2 \mathrm{~K}$. Thus, the need for titanification of cavities is found to be the single most necessary step in the preparation of high frequency cavities resulting in improved performance through enhanced phonon peak and significantly increased Kapitza conductance.

\section{B. Effect of Kapitza conductance}

The effect of the large surface index (increased surface area) on Kapitza conductance remained inconclusive possibly due to other effects influencing the heat transfer. These include, but are not limited to, the effect of surface strains, BCP etching, or the distribution of crystal orientation on the surface on the Kapitza conductance. Although, both the low as well as the moderate temperature annealing has shown a consistent increase in Kapitza conductance, however, the exact cause of this increase is still undetermined. More research in this direction is needed to find the exact cause of increase in Kapitza conductance after annealing.

\section{Looking ahead: Large phonon peak and large Kapitza conductance}

About a decade ago, the role of the thermal parameters seemed to be exhaustively investigated and well known to the SRF community. Not much room for improvement in the performance of SRF cavity was talked about through improvement in its thermal parameters. In this study, effort has been made to change this view by opening up new challenges for further work in this direction.

Enhancement of the phonon peak in polycrystalline $\mathrm{Nb}$ could possibly be realizable either through high temperature $\left(1800\right.$ to $\left.1900^{\circ} \mathrm{C}\right)$ annealing or for a longer duration at moderate temperatures. The use of unstrained single crystal/large grain $\mathrm{Nb}$ also seems to be a promising material to look for a considerably more enhanced phonon peak. Sometimes the lattice imperfections even in these single crystal/large grain material may not show the enhanced phonon peak. Moderate temperature annealing of such material may be necessary to bring the phonon peak to prominence.

Similarly, the domain of Kapitza conductance still poses many unanswered questions for which further research is needed. As discussed earlier, the effect of surface strains and/or crystal orientations on the surface need more attention to understand the effect of increased Kapitza conductance, as seen in this study through titanification process. 
[1] H. Padamsee, J. Knobloch, and T. Hays, RF Superconductivity for Accelerators (Wiley, New York, 1998).

[2] J. Halbritter, in Proceedings of the 2nd Workshop on RF Superconductivity, CERN, Geneva, Switzerland, 1984, edited by H. Lengeler (CERN, Geneva, 1984), p. 427 [http://cdsweb.cern.ch/record/114742/holdings?ln=en].

[3] F. Palmer, in Proceedings of the 3rd Workshop on RF Superconductivity, edited by K. W. Shepard (Argonne National Laboratory, Argonne, IL, 1988), p. 309.

[4] J.P. Turneasure, in Proceedings of the 1972 Applied Superconducting Conference, Annapolis (IEEE, New York, 1972), p. 621.

[5] J. Amrit, C.Z. Antoine, M.X. Francois, and H. Safa, in Advances in Cryogenic Engineering: Proceedings of the
Cryogenic Engineering Conference, 2002 (AIP, New York, 2002), Vol. 613, pp. 499-506.

[6] A. Aizaz and T. L. Grimm, in Advances in Cryogenic Engineering: Transactions of the Cryogenic Engineering Conference, edited by J. Weisend II (AIP, New York, 2006), Vol. 823, pp. 1179-1186.

[7] R. L. Geng (private communication).

[8] J. Amrit and M. X. Francois, J. Low Temp. Phys. 128, 113 (2002).

[9] J. Amrit, Superlattices and Microstructures (Elsevier Science Publisher, New York, 2004), Vol. 35, pp. 187-194.

[10] S. Bousson, M. Fouaidy, T. Junquea, N. Hammoudi, J. C. Le Scornet, and J. Lesrel, in Proceedings of 9th Workshop on RF Superconductivity, 1999. 